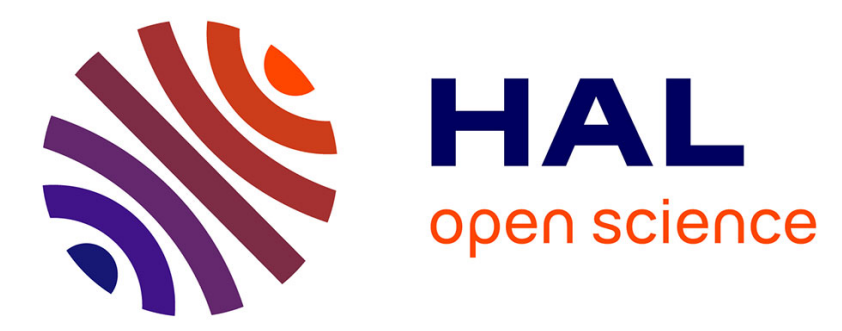

\title{
On the Interest of Bulk Conductivity Measurements for Hydraulic Dispersivity Estimation from Miscible Displacement Experiments in Rock Samples
}

Alexis Maineult, Jean-Baptiste Clavaud, Maria Zamora

\section{- To cite this version:}

Alexis Maineult, Jean-Baptiste Clavaud, Maria Zamora. On the Interest of Bulk Conductivity Measurements for Hydraulic Dispersivity Estimation from Miscible Displacement Experiments in Rock Samples. Transport in Porous Media, 2016, 115 (1), pp.21-34. 10.1007/s11242-016-0749-0 . hal01372008

\section{HAL Id: hal-01372008 https://hal.sorbonne-universite.fr/hal-01372008}

Submitted on 26 Sep 2016

HAL is a multi-disciplinary open access archive for the deposit and dissemination of scientific research documents, whether they are published or not. The documents may come from teaching and research institutions in France or abroad, or from public or private research centers.
L'archive ouverte pluridisciplinaire HAL, est destinée au dépôt et à la diffusion de documents scientifiques de niveau recherche, publiés ou non, émanant des établissements d'enseignement et de recherche français ou étrangers, des laboratoires publics ou privés. 
3 On the interest of bulk conductivity measurements for hydraulic dispersivity estimation

4 from miscible displacement experiments in rock samples.

6 Alexis Maineult ${ }^{1,2,+}$, Jean-Baptiste Clavaud ${ }^{1, *}$ and Maria Zamora ${ }^{1}$

8 1) Institut de Physique du Globe de Paris, Sorbonne Paris Cité, Univ Paris Diderot, UMR 7154

9 CNRS, 1 rue Jussieu, 75005 Paris, France

10 2) Sorbonne Universités, UPMC Univ Paris 06, CNRS, EPHE, UMR 7619 Metis, 4 place

11 Jussieu, 75005 Paris, France

$12{ }^{*}$ now at Chevron Energy Technology Company, 1500 Louisiana St., Houston, TX 77002, USA

$15+$ corresponding author. Phone: $+33(0) 1442743$ 36. E-mail address: alexis.maineult@upmc.fr 


\section{SUMMARY}

18 The determination of the hydraulic dispersivity and effective fraction of porous medium

19 contributing to transport on soil and rock sample in the laboratory is important to understand and

20 model the evolution of miscible contaminant plumes in groundwater. Classical methods are based

21 on the interpretation of the breakthrough curve, i.e., the evolution of the concentration in

22 contaminant at the downstream end-face of a sample into which a front of contaminant is

23 advected. Here we present an experimental device aimed at performing such measurements, but

24 also allowing the bulk electrical conductivity of the sample to be measured. We show that the

25 dispersivity and effective fraction can be inferred from this electrical measurement, and that the

26 combined use of both out-flowing fluid conductivity and bulk conductivity allows the incertitude

27 on the dispersivity and effective fraction to be significantly enhanced.

28

29 Keywords: miscible displacement, hydraulic dispersivity, breakthrough curve, electrical

30 conductivity 


\section{Introduction}

Miscible contaminants flowing through a porous medium by advection are mixing with

35 the non-contaminated water, yielding to dilution of the plume. This so-called dispersion

36 phenomenon originates from the fact that the fluid moves faster in larger pores than in smaller

37 ones and faster in the centres of the pores than along the walls, and also that some pathways are

38 longer than others (e.g., Fetter 2001). If we consider an isotropic and homogeneous soil or rock

39 sample, and assume that i) the flow through it is purely one-dimensional and ii) the dispersion

40 process is Fickian, the concentration field $C(x, t)$ (in $\mathrm{mol} \mathrm{m}^{-3}$ ) inside the sample, where $x$ (in $\mathrm{m}$ )

41 stands for the distance from the upstream-face of the sample and $t$ (in $\mathrm{s}$ ) for the elapsed time,

42 obeys the transport equation:

43

$$
\frac{\partial C}{\partial t}=D \frac{\partial^{2} C}{\partial x^{2}}-v \frac{\partial C}{\partial x},
$$

44 Here $D$ is the hydrodynamic dispersion coefficient (in $\mathrm{m}^{2} \mathrm{~s}^{-1}$ ) and $v$ the linear average velocity of

45 water flowing through the sample (in $\mathrm{m} \mathrm{s}^{-1}$ ). Provided that diffusion processes can be neglected,

46 the dispersion coefficient in Eq. (1) can be written $D=\lambda v$, where $\lambda$ is the hydraulic dispersivity

47 (in m). Even though the dispersivity changes with the observation scale (e.g., Xu and Epstein

48 1995), it is often required to determine its value on soil or rock samples to understand and model

49 the dynamics of contaminants at the field scale.

50 The classical way to perform such measurements in the laboratory consists in applying a

51 straight front of salty solution at the entrance (i.e., the upstream face) of the sample subjected to

52 permanent, laminar flow, and to measure the concentration at the outlet (i.e., the downstream

53 face). The evolution of the concentration of the out-flowing fluid, or "breakthrough curve", is

54 generally sigmoidal, provided that the medium is homogeneous at the scale of the sample and 
55 that no other processes occur, such as chemical reactions with the minerals. The spreading of the 56 curve increases with the dispersivity. Knowing the theoretical formulation of the breakthrough

57 curve, it is then possible to determine the value of the dispersivity by fitting methods.

58 The out-flowing concentration is an integrated value, which presents variations only when

59 the salt front reaches the downstream face of the sample. Other methods, such as electrical

60 measurements, can give information as soon as the salt front penetrates the sample. For example,

61 Odling et al. (2007) used electrical impedance measurements on microfractured granite to

62 determine its longitudinal dispersivity. They showed that such a technique provided supplemental

63 information about the dispersion process inside the sample. Here we report on an apparatus

64 allowing the bulk conductivity of a rock sample to be measured during miscible displacement

65 experiments. We show that fitting the classical breakthrough curve to the measured electrical

66 conductivity of the out-flowing fluid, combined to fitting the theoretical evolution of the bulk

67 conductivity of the sample to the measured one, enables a more accurate determination of the

68 hydraulic dispersivity and fraction of the porous volume effectively contributing to the transport.

70 2. Materials and method

\section{2.1. Device and procedure}

73 We devised, constructed and tested an experimental set-up (Fig. 1), aimed at measuring

74 the bulk electrical conductivity $\sigma_{b}$ (in $\mathrm{S} \mathrm{m}^{-1}$ ) of large-sized samples (i.e., about 5 to $10 \mathrm{~cm}$ in

75 diameter and $10-40 \mathrm{~cm}$ or more in length), as well as the electrical conductivity of the fluid that is

76 out-flowing from the sample, denoted $\sigma_{f}$. This device is usable to perform miscible displacement

77 experiments and therefore infer the hydraulic dispersivity $\lambda$ (in $\mathrm{m}$ ) and the effective fraction of 
78 the porous volume contributing to transport, denoted $f$ (no unit).

The upstream and downstream end-faces of the sample, which is placed horizontally to

80 avoid the influence of gravity, are connected to a hydraulic circuit. They are also in perfect

81 contact with stainless steel electrodes, whose external side is protected by a PVC cap to ensure

82 electrical isolation. The conductivity of the out-flowing fluid is measured with a custom-made

83 cell with four platinum electrodes that was calibrated with various brines at different frequencies.

84 The fluid conductivity and the bulk conductivity of the sample are measured with an impedance-

85 meter HP 4263A, at the frequencies of 120 and $1000 \mathrm{~Hz}$. The optimal frequency, which

86 minimizes the polarization effects, is equal to $1 \mathrm{kHz}$. Note that concerning the bulk conductivity,

87 the measure is done with the two electrodes at the end-faces of the sample only, each electrode

88 serving for both current injection and potential measurement.

89 To check its watertightness, the sample is first put under vacuum for a few hours. The

90 sample is then saturated with distilled and de-aerated water. The porosity accessible by water

91 under vacuum, which is close to the connected porosity, is deduced from the amount of injected

92 water required to fill the connected voids, with an accuracy comprised between 0.3 and 3.5

93 percents. Then we let the fluid flowing through the sample from the upstream reservoir filled

94 with distilled water. Once the chemical equilibrium is reached, i.e. when the conductivity of the

95 out-flowing fluid is constant, the distilled water saturating the sample is flushed by $\mathrm{NaCl}$ brine at

96 a concentration around $3 \mathrm{~g} \mathrm{~L}^{-1}$, coming from the brine reservoir. This value of the concentration

97 is large enough to consider the contribution of the surface conduction to the total electrical

98 conductivity negligible, but small enough to neglect density-driven flow, at least for highly

99 permeable samples. Brine is injected until the out-flowing solution has the same electrical

100 conductivity. In practice, a given amount of brine is introduced in the sample. The electrical

101 measurements are carried out rapidly enough to neglect the effects of pure diffusion and reduce 
102 the density effects, if any. Afterwards we proceed to a new injection step, and so on.

103

$104 \quad 2.2$ Tested sample

105 To test our approach, we use a decimetric core of Saint-Maximin limestone (denoted

106 SML). It is a bioclastic limestone, having a large macro-porosity, and a small amount of

107 intraclastic porosity (Fig. 2a). The results of mercury injection carried out on a centimetric core

108 (Fig. 2b) show that the distribution of the pore access radii is bi-modal with to modes at 13.6 and

$1090.13 \mu \mathrm{m}$ respectively. However, the second mode, which corresponds to the intraclastic porosity,

110 is clearly dominated by the first mode.

111 The sample was $309 \mathrm{~mm}$ in length, with a diameter of $97.90 \mathrm{~mm}$. The volume of the

112 sample $\left(2326 \mathrm{~cm}^{3}\right)$ is therefore two orders of magnitude larger than the volumes of rock samples

113 commonly studied in the laboratory for permeability estimation (i.e., 20 to $50 \mathrm{~cm}^{3}$ ). This size was

114 chosen to acquire measurements at a scale significantly larger than the representative elementary

115 volume (e.g., Henriette et al. 1989). After machining, the sample was dried in an oven at $60{ }^{\circ} \mathrm{C}$

116 for several days, until its weight stabilized. Then the lateral surface was waterproofed by

117 covering it with epoxy resin. The connected porosity value, determined from weighting, was

118 equal to $36.6 \pm 0.5 \%$. From flow-rate measurement, the water permeability was estimated to be

$1191718 \pm 42 \mathrm{mD}$.

120 To check if advection is predominant over diffusion in our experiment, we estimated the

121 Péclet number Pe. The classical formulation $\mathrm{Pe}=v_{\mathrm{DF}} d / D_{m}$ (e.g., Sahimi 1993), where $v_{\mathrm{DF}}$ is the

122 Dupuit-Forcheimer velocity (in $\mathrm{m} \mathrm{s}^{-1}$ ), $d$ the mean grain diameter (in $\mathrm{m}$ ) and $D_{m}$ the molecular

123 diffusion coefficient $\left(1.510^{-9} \mathrm{~m}^{2} \mathrm{~s}^{-1}\right.$ for $\left.\mathrm{NaCl}\right)$, is not applicable for non-granular rocks.

124 Therefore, we used the non-correlation radius $r_{n c}$, estimated from image analysis, instead of $d / 2$. 
125 This yields to $\mathrm{Pe}=2 v_{\mathrm{DF}} r_{n c} / D_{m}$. For the highly permeable sample SML, $v_{D F}$ was equal to 0.35

$126 \mathrm{~mm} \mathrm{~s}^{-1}$ during the experiment, and $r_{n c}$ was estimated to $175 \mu \mathrm{m}$. The Péclet number is thus

127 around 80, meaning that in this case advection can be neglected (power-law regime, e.g. Sahimi 128 1993).

129 For the miscible displacement experiment, the conductivity of the initial fluid after

130 stabilization was equal to $4.7 \mathrm{mS} \mathrm{m}^{-1}$. The conductivity of the injected fluid was $540 \mathrm{mS} \mathrm{m}^{-1}$.

131 (i.e., $54.3 \mathrm{mmol} \mathrm{L}^{-1}$ or $3.17 \mathrm{~g} \mathrm{~L}^{-1} \mathrm{NaCl}$ ). The bulk conductivity of the sample was equal to 1.4

$132 \mathrm{mS} \mathrm{m}^{-1}$ initially, and to $90.1 \mathrm{mS} \mathrm{m}^{-1}$ at the end of the experiment, yielding to an electrical

133 formation factor $F=\sigma_{f} / \sigma_{b}$ equal to 6.

\section{3. Theory}

\section{3.1. Problem and solution for a $1 D$ isotropic and homogeneous medium}

138 Considering that Eq. (1) applies to our case, the initial condition is $C(x, 0)=C_{0}$, where $C_{0}$ 139 is the equivalent salt concentration of the solution initially saturating the pore space. The 140 upstream boundary condition, corresponding to the injection of brine starting at $t=0$, can be 141 formalized as $C(0, t)=C_{0}+\left(C_{\text {brine }}-C_{0}\right) \mathrm{H}(t)$ where $C_{\text {brine }}$ is the concentration of the brine and $\mathrm{H}$ 142 the Heaviside step function. The solution is given by (e.g., Ogata and Banks 1961; Fried and

143 Combarnous 1971; Gupta and Greenkorn 1974; Basak and Murty 1979):

$$
C(x, t)=C_{0}+\frac{C_{\text {brine }}-C_{0}}{2}\left[\operatorname{erfc}\left(\frac{x-v t}{2 \sqrt{D t}}\right)+\exp \left(\frac{v x}{D}\right) \operatorname{erfc}\left(\frac{x+v t}{2 \sqrt{D t}}\right)\right],
$$

145 where erfc is the complementary error function. The second term in the brackets has an influence 146 only if $t$ is close to 0, and can be neglected otherwise (e.g., Ogata and Banks 1961; Pfannkuch 147 1963), yielding to the following approximation of Eq. (2): 


$$
C(x, t) \approx C_{0}+\frac{C_{\text {brine }}-C_{0}}{2} \operatorname{erfc}\left(\frac{x-v t}{2 \sqrt{D t}}\right)
$$

\subsection{Non-dimensionalization of the solution}

152 to use the normalized variation of the concentration $\delta C$ (no unit) defined by

$$
\delta C(x, t)=\frac{C(x, t)-C_{0}}{C_{\text {brine }}-C_{0}} .
$$

154 Since the injection is not continuous but performed step by step (see end of section 2.1), it is

155 convenient to consider $\delta C$ as a function of the normalized injected volume of brine $V_{i}^{n}$, expressed

156 in pore volumes, as (e.g., Pickens and Grisak 1981):

$$
V_{i}^{n}=\frac{V_{i}}{V_{p c}}
$$

158 where $V_{i}$ is the injected volume of brine and $V_{p c}$ the total connected porous volume of the sample,

159 equal to the total porosity times the volume of the sample. Following this way, the curves $\delta C$

160 versus $V_{i}^{n}$ for different samples are similarly scaled and therefore directly comparable. Indeed,

161 the time required for the injection of a given volume of brine, which depends on the permeability

162 of the rock, is eliminated from Eq. (3): considering that $V_{i}^{n}$ is also equal to $v t / L$ where $L$ is the

163 length of the sample (in $\mathrm{m}$ ), and assuming that the molecular diffusion is negligible so that we

164 can introduce the hydraulic dispersivity as $\lambda=D / v$, the combination of Eqs. (3) and (4) leads to:

$$
\delta C(x, t) \approx \frac{1}{2} \operatorname{erfc}\left(\frac{1}{2} \sqrt{\frac{L}{\lambda}} \frac{\left(\frac{x}{L}-V_{i}^{n}\right)}{\sqrt{V_{i}^{n}}}\right) .
$$




$$
\delta C_{\text {out }}(t)=\delta C(L, t) \approx \frac{1}{2} \operatorname{erfc}\left(\frac{1}{2} \sqrt{\frac{L}{\lambda}} \frac{1-V_{i}^{n}}{\sqrt{V_{i}^{n}}}\right)
$$

169 Classically, the $\lambda / L$ ratio is inferred from the measured values of $\delta C_{\text {out }}$ (i.e., the normalized

170 breakthrough curve) by fitting the theoretical curve (Eq. (7)) to them. Note here that this

171 theoretical curve passes through the point $\mathrm{M}_{1,0.5}=\left(V_{i}^{n}, \delta C_{\text {out }}\right)=(1,0.5)$, since the volume of brine

172 required for the centre of mass of the front to arrive at the downstream end of the sample is equal

173 to one pore volume.

174 It is assumed here that the whole connected pore volume contributes to the transport, but

175 it is rarely expected. For example, dead-end pores do not contribute to the transport processes. If

176 the pore volume contributing to transport (or "efficient porosity"), denoted $V_{p c}{ }^{*}$, is smaller than

$177 V_{p c}$, the experimental curve $\delta C_{\text {out }}$ versus $V_{i}^{n}$ is shifted to the left. In other words, the injected

178 volume, normalized using Eq. (5), for which $\delta C_{\text {out }}$ is equal to 0.5 , is smaller than 1 . In this case,

179 one should also find the best $V_{p c}{ }^{*}$ to be used in place of $V_{p c}$ in Eq. (5). To do so, we define the

180 effective fraction of the porous volume contributing to the transport as $f=V_{p c}{ }^{*} / V_{p c}$. Equation (5)

181 must thus be rewritten as:

$$
V_{i}^{n}=\frac{V_{i}}{V_{p c}{ }^{*}}=\frac{V_{i}}{f V_{p c}} .
$$

183 The problem then involves two parameters, $\lambda / L$ and $f$, to be found using Eqs. (7) and (8).

It should be noted here that if no theoretical curve can explain the data, it means that the

185 medium is not homogeneous, and/or that retardation processes occur such as trapping in small

186 pores, or that molecular diffusion is not negligible, or that density-driven flow occurs, or even

187 chemical reactions with the reactive mineral phases happen. 
189 corrected from the volume of the tube between the T-valve (Fig. 1) and the upstream end of the 190 sample for the bulk sample conductivity, and from the same volume plus the volume of the tube

191 between the downstream end of the sample and the conductivity cell for the conductivity of the 192 out-flowing fluid.

\subsection{Conversion of conductivity to concentration}

As previously stated, we do not measure the salt concentration directly, but the electrical

196 conductivity of the fluid. To transform the concentration $C$ into conductivity $\sigma_{f}$, and reciprocally

$197 \sigma_{f}$ into $C$, we use the empirical relations established by Sen and Goode (1992) for NaCl brine:

$$
\sigma_{f}=\left(5.6+0.27 T-1.510^{-4} T^{2}\right) M-\frac{2.36+0.099 T}{1+0.214 M} M^{\frac{3}{2}}
$$

where $T$ is the temperature (in ${ }^{\circ} \mathrm{C}$ ) and $M$ is the molality (in $\mathrm{mol} \mathrm{kg}^{-1}$ ). To convert the

200 concentration $C_{f}$ into molality, we use the CRC Handbook Table at $20^{\circ} \mathrm{C}$ (Lide 2008).

$$
\delta \sigma_{\text {out }}(t)=\frac{\sigma_{\text {out }}(t)-\sigma_{\text {out }, 0}}{\sigma_{\text {out } \max }-\sigma_{\text {out }, 0}}
$$

203 where $\sigma_{\text {out }, 0}$ and $\sigma_{\text {out,max }}$ are the initial and maximal (i.e., the plateau value at the end of the 204 experiment) conductivities of the effluent solution, respectively. We proceeded similarly for the 205 bulk conductivity of the sample $\sigma_{b}$ :

$$
\delta \sigma_{b}(t)=\frac{\sigma_{b}(t)-\sigma_{b, 0}}{\sigma_{b, \max }-\sigma_{b, 0}}
$$

207 where $\sigma_{b, 0}$ is the initial and $\sigma_{b, \max }$ the maximal (i.e., the plateau value at the end of the experiment) value of the bulk conductivity. 


\section{4. Estimation of the dispersivity}

211 To estimate the dispersivity $\lambda$, we first use the classical method, which consists in fitting a

212 straight line in Henry's space to the experimental points of the out-flowing fluid concentration.

213 Secondly we systematically explored the parameter space. This second method allows the

214 effective fraction $f$ to be taken into account, and the sample conductivity to be also considered.

\section{4.1. Cost-function}

217 To evaluate the agreement between the data (i.e., the normalized measured conductivity 218 variations) and the model (i.e., the normalized predicted conductivity variations), a cost-function

$219 R$ as meaningful as possible has to be defined. We used the determination coefficient $r^{2}$, which 220 quantifies the mean deviation of the $N$ experimental points (vector $\mathbf{d}^{\text {obs }}$ ) from the predictions

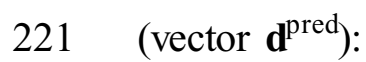

$$
r^{2}=1-\frac{\sum_{i=1}^{N}\left(d_{i}^{\mathrm{obs}}-d_{i}^{\mathrm{pred}}\right)^{2}}{\sum_{i=1}^{N}\left(d^{\mathrm{obs}}-\left\langle\mathbf{d}^{\mathrm{obs}}\right\rangle\right)^{2}}
$$

223 Moreover, considering that the concentration break-through curves (Eq. (7)) are similar to

224 cumulative Gaussian distributions, we combined $r^{2}$ with the Kolmogorov-Smirnov (KS) test (e.g., 225 Press et al. 1997), usually used as an estimator of the semblance between two distributions, given 226 by:

$$
\left\{\begin{array}{l}
\mu=\left(\sqrt{N}+0.12+\frac{0.11}{\sqrt{N}}\right) \max \left|\mathbf{d}^{\text {pred }}-\mathbf{d}^{o b s}\right| \\
K S=2 \sum_{j=1}^{\infty}(-1)^{j-1} e^{-2 j^{2} \mu^{2}}
\end{array}\right.
$$


228 We thus used the function $R=K S r^{2}$; an optimal fit is obtained when $R=1$.

\subsection{Fitting method in Henry's space}

231

232

Defining the two parameters $\alpha$ (no unit) and $\Gamma$ (no unit) as:

$$
\left\{\begin{array}{l}
\alpha=\frac{1}{2} \sqrt{\frac{L}{\lambda}} \\
\Gamma=\frac{1-V_{i}^{n}}{\sqrt{V_{i}^{n}}}
\end{array}\right.
$$

233 Eq. (7) can be rewritten as:

234

$$
\delta C_{\text {out }}(t)=\frac{1}{2} \operatorname{erfc}(\alpha \Gamma)
$$

235 Therefore the curve $\Gamma$ versus $\delta C_{\text {out }}$ plotted in linear arithmetic probability paper (in ordinates)

236 forms a straight line (e.g., Brigham 1974), also called Henry's line. The slope of this line,

237 denoted $p$, is theoretically given by:

$$
p=-\frac{1}{\alpha \sqrt{2}}=-\sqrt{2} \sqrt{\frac{\lambda}{L}} .
$$

239 We did not apply the classical two-point Taylor's (1953) method to determine $\lambda$ (e.g., Fried and

240 Combarnous 1971; Gupta and Greenkorn 1974; Pickens and Grisak 1981). Instead, we

241 determined the straight line fitting the data in the least-square sense, as suggested by Gupta and

242 Greenkorn (1974), considering that this method provides more accurate values for the slope $p$.

243 Note that in this case it is necessary to restrict the data to the interval for which the conductivity

244 variation is linear, i.e., generally for $\delta C_{\text {out }}$ comprised between 0.1 and 0.9 . The dispersivity is then

245 deduced using Eq. (16) as $\lambda=L p^{2} / 2$.

246 The observed normalized conductivity $\delta \sigma_{\text {out }}$ and deduced normalized concentration $\delta C_{\text {out }}$ 247 of the out-flowing fluid as a function of the volume of injected brine $V_{i}^{n}$, expressed in fraction of 
248 porous volume, are shown in Fig. 3a for SML sample. The concentrations $\delta C_{\text {out }}$ are distributed

249 along a sigmoid, which seems to pass very close to the point $\mathrm{M}_{1,0.5}=\left(V_{i}^{n}, \delta C_{\text {out }}\right)=(1,0.5)$,

250 meaning that the whole pore volume contribute to the transport. The associated $\Gamma$ values

251 computed using Eq. (14), versus normalized experimental concentration $\delta C_{\text {out }}$, are distributed

252 along a straight line (Fig. 3b). The line fitting the best the data, in the least-squares sense, passes

253 through the point $\left(\delta C_{o u t}, \Gamma\right)=(0.5,0)$, meaning that indeed the effective fraction $f$ is equal to 1 .

254 The slope of the fitting line gives a value of $4.018 \mathrm{~mm}$ for the dispersivity $\lambda_{H L}$. The modelled

255 curves $\delta C_{\text {out }}$ (continuous line in Fig. 3a, obtained with Eqs. (7) and (5)) and $\delta \sigma_{\text {out }}$ (dotted line)

256 versus the volume of injected brine, expressed in fraction of porous volume, explain the data

257 rather well (the cost-function $R^{H L}$ is close to 1.0).

258

259 4.3. Parameter space exploration

260 The previous procedure takes into account the conductivity variation of the out-flowing

261 fluid only. Moreover, it does not provide a confidence interval for the dispersivity or the effective

262 fraction of porous volume. We hereafter estimate the dispersivity directly from the curves of the

263 out-flowing fluid conductivity variations $\delta \sigma_{o u t}$ or/and the variations of the bulk conductivity $\delta \sigma_{b}$

264 versus the injected volume $V_{i}^{n}$ by exploring the parameter space

265 To estimate the sample bulk conductivity $\sigma_{b}$, we apply the generalized Reuss average,

266 since the measurement is performed in the direction of the concentration gradient. We assume

267 that i) the concentration front is one-dimensional along the axis of the sample (i.e., the sample is

268 homogeneous and the front is straight, i.e., the density effect are negligible), and ii) the formation

269 factor $F$ does not depend on the value of the local fluid conductivity. Therefore, $\sigma_{b}$ can be written

270 as (Odling et al. 2007): 


$$
\frac{1}{\sigma_{b}(t)}=\frac{F}{L} \int_{0}^{L} \frac{d x}{\sigma_{f}(C(x, t))}
$$

272 where $\sigma_{f}(C(x, t))$ corresponds to the fluid conductivity distribution inside the sample (computed

273 using Eqs. (3) and (9)). It is important to note that the knowledge of the formation factor is not

274 required, since it vanishes in the computation of the normalized variation of the bulk conductivity 275 using Eq. (11).

276 The application to limestone SML is shown in Figs. 4, 5 and 6. We explored the 277 dispersivity values in the interval comprised between 0 and $20 \mathrm{~mm}$ (based on the value 278 previously determined for $\lambda_{H L}$, ) with a spacing of $0.1 \mathrm{~mm}$, and the values of the effective fraction 279 in the interval comprised between 0.85 and 1 with a spacing of 0.001 .

280 When the exploration method is applied to the fluid conductivity only (Fig. 4a), the 281 maximal value of the cost-function $R_{\max }^{f}$ is equal to 0.99951 (with a Kolmogorov-Smirnov value 282 equal to $100 \%$ ) and corresponds to a dispersivity $\lambda_{f}$ (subscript "f" standing for "fluid") of $4.0 \mathrm{~mm}$ 283 and an effective fraction $f_{f}$ of 0.982 . The value of the dispersivity is very close to the value 284 determined previously using Henry's line $(4.018 \mathrm{~mm})$. The value of the effective fraction is very 285 close to 1 , but is slightly smaller. The predictions computed with Eqs. (7), (8) and (10) explain 286 very well the data (Fig. 4b).

287 We proceeded similarly for the bulk conductivity only (Fig. 5). As for the fluid, the 288 normalized bulk conductivity of the sample is sigmoidal, but the bulk conductivity evolves as 289 soon as brine is injected in the sample (Fig. 5b). The dispersivity $\lambda_{s}$ (subscript "s" standing for 290 "sample") is equal to $3.3 \mathrm{~mm}$, a value slightly smaller than $\lambda_{f}$. The effective fraction $f_{s}$ is equal to 291 0.987. The prediction, computed using Eqs. (3), (9), (11) and (17), explains here again rather well 292 the data, with a cost-function $R_{\max }^{s}$ equal to 0.99954 . However, it seems that the dispersivity may 
293 be slightly underestimated for injected volume above 1, the prediction being systematically

294 higher than the experimental points.

295 We finally apply the same methodology to find the best dispersivity and effective fraction

296 explaining both fluid and sample conductivities simultaneously (Fig. 6). In this case, we defined

297 the cost-function as the product of the two cost-functions for the fluid and the sample, i.e.

$298 R=R^{f} R^{s}$. Doing so, the best dispersivity value $\lambda$ is equal to $3.5 \mathrm{~mm}$ and the best effective

299 fraction $f$ to 0.986 (Fig. 6a). The predictions explained rather well the fluid conductivity (Fig. 6b)

300 and the bulk conductivity of the sample (Fig. 6c).

301

\section{5. Discussion}

303 First of all, a coherent estimation of the dispersivity and of the effective fraction is

304 provided by the interpretation of the evolution of the bulk conductivity of the sample.

305 Secondly, when performing sensitivity analysis, it can be seen that the ranges of

306 dispersivity and effective fraction which produce high values of the cost-function for the out-

307 flowing fluid conductivity are quite large (red area in Fig. 4a). For instance, values of dispersivity

308 between 1.5 and 8.1 and effective fraction between 0.941 and 1 produce a cost-function value

309 superior to 0.99 , sufficiently high to produce satisfactorily predictions. A way to estimate the

310 error interval on the determined values of dispersivity and effective fraction may be to consider

311 their values which produce cost-function values higher than a certain threshold value. Here we

312 can consider $R^{f}=0.998$. In this case, the dispersivity $\lambda_{f}$ is comprised between 3 and 5.4 , and the

313 effective fraction $f_{f}$ between 0.966 and 0.998 . From the exploration of the parameter space for the

314 bulk conductivity of the sample, it can be concluded that the bulk conductivity is more sensitive

315 to the variations of the dispersivity (the red area in Fig. 5a is less extended in the $\lambda$-direction than 
316 in Fig. 4a), but is less sensitive to the variations of the effective fraction (the red area is more

317 extended in the $f$-direction). Considering again the threshold value of 0.998 for $R^{S}$, the

318 dispersivity $\lambda_{s}$ is comprised between 2.2 and 4.5, and the effective fraction $f_{f}$ between 0.955 and

319 1. The dispersivity is thus best determined by the bulk conductivity and the effective fraction by

320 the out-flowing fluid conductivity. This illustrates the fact that using the bulk conductivity of the

321 sample provides supplemental information, compared to the use of the out-flowing fluid

322 conductivity alone. Finally, when considering the exploration on both out-flowing fluid

323 conductivity and bulk conductivity of the sample, the area producing high values of the cost-

324 function $R$ is much restrained than in the previous two cases. Indeed, the $R=0.998$ threshold

325 gives an interval of [3.2,3.9] for the dispersivity $\lambda$ and an interval of [0.978,0.994] for the

326 effective fraction $f$.

327 The fact that the effective fraction is not equal to 1 , but close to it, means that a small part

328 of the porous volume does not contribute to the transport. This non-contributing volume may be

329 the intraclastic porosity associated with the second mode in the pore access radius distribution

330 (Fig. 2b). Moreover, the fact that the predictions are slightly higher than the observations for

331 injected volume greater than 1 (Figs. $4 b, 5 b$ and $6 b c$ ) may be results from retardation processes of

332 small intensity - maybe the penetration of salt in this intraclastic porosity.

333 Finally, the fact that all the estimated dispersivities $\lambda_{H L}, \lambda_{f}, \lambda_{s}$ and $\lambda$, as well as the

334 effective fractions $f_{f}, f_{s}$ and $f$ are close one to the other, and that the data are rather well explained

335 by these values, means that all the assumptions made here (mainly: the sample is homogeneous

336 and the diffusion and the density effect can be neglected) are reasonable. For less permeable

337 samples with lower Péclet number, and/or for higher brine concentration, more complicated

338 models have to be implemented, but this is beyond the scope of this study. 


\section{6. Conclusions}

341 We added to the classical measurement of the breakthrough curve (evolution of the

342 conductivity of the out-flowing fluid) during miscible displacements experiments the

343 measurement of the evolution of the bulk conductivity of the sample. A methodology based on

344 the exploration of the possible values for the hydraulic dispersivity and for the effective fraction

345 of total connected porous volume contributing to the transport was developed, which also provide

346 confidence intervals The values obtained on a sample of limestone using the out-flowing fluid

347 conductivity only or the bulk conductivity of the sample only are coherent with those determined

348 by the classical method of Henry line. The most interesting point is that the combined use of out-

349 flowing fluid and bulk conductivities also produces coherent values, but with significantly

350 reduced confidence intervals. This underlines the interest of systematically including the

351 measurement of the bulk conductivity during laboratory measurements of the dispersivity on rock

352 samples.

353

\section{Acknowledgments}

355 The authors thank all the anonymous reviewers of this work for their helpful comments.

356 This is IPGP contribution $n^{\circ} 3744$.

\section{$358 \quad$ References}

359 Basak, P., Murty, V.V.N.: Determination of hydrodynamic dispersion coefficients using

360 "inverfc". J. Hydrol. 41, 43-48 (1979)

361 Brigham, W.E.: Mixing equations in short laboratory columns. Soc. Pet. Eng. J. 14, 91-99 (1974)

362 Fetter, C.W.: Applied Hydrogeolgy, $4^{\text {th }}$ Edition. Prentice Hall, NJ (2001) 
363 Fried, J.J., Combarnous, M.A.: Dispersion in porous media. Adv. Hydrosci. 7, 169-282 (1971).

364 Gupta, S.P., Greenkorn, R.A.: Determination of dispersion and nonlinear adsorption parameters

365 for flow in porous media. Water. Resources Res. 10, 839-846 (1974)

366 Henriette, A., Jacquin, C.G., Adler, P.M.: The effective permeability of heterogeneous porous

367 media. Physicochem. Hydrodyn. 11, 63-80 (1989)

368 Lide, D.R. (ed): Handbook of chemistry and physics, $89^{\text {th }}$ edition. CRC Press, Boca Raton, FL

$369 \quad(2008)$

370 Odling, N.W.A., Elphick, S.C., Meredith, P., Main, I., Ngwenya, B.T.: Laboratory measurement

371 of hydrodynamic saline dispersion within a micro-fracture network induced in granite. Earth

372 Planet. Sci. Lett. 260, 407-418 (2007)

373 Ogata, A., Banks, R.B.: A solution of the differential equation of longitudinal dispersion in

374 porous media. U.S. Geol. Surv. Professionnal Paper, 411-A (1961)

375 Pfannkuch, H.O.: Contribution à l'étude des déplacements de fluides miscibles dans un milieu

376 poreux. Rev. Inst. Fr. Pét. 18, 215-270 (1963)

377 Pickens, J.F., Grisak, G.E.: Scale-dependent dispersion in a stratified granular aquifer. Water

378 Resour. Res. 17, 1191-1211 (1981)

379 Press, W.H., Teukolsky, S.A., Vetterling, W.T., Flannery, B.P.: Numerical Recipes in C: the Art 380 of Scientific Computing, $2^{\text {nd }}$ Edition. Cambridge Univ. Press, New York (1992)

381 Sahimi, M.: Flow phenomena in rocks: from continuum models to fractals, percolation, cellular 382 automata, and simulated annealing. Rev. Modern Phys. 65, 1393-1534 (1993)

383 Sen, P.N., Goode, P.A.: Influence of temperature on electrical conductivity of shaly sands.

384 Geophysics 57, 89-96 (1992)

385 Six, P.: Contribution à l'étude de la perméabilité d'une roche poreuse à un liquide. Rev. Inst. Fr.

386 Pét. 17, 1454-1472 (1962) 
387 Taylor, G.: Dispersion of soluble matter in solvent flowing slowly through a tube. Proc. Royal. 388 Soc. Ser. A 219, 186-203 (1953)

389 Xu, M.J., Eckstein, Y.: Use of weighted least-squares method in evaluation of the relationship 390 between dispersivity and field-scale. Ground Water 33, 905-908 (1995) 


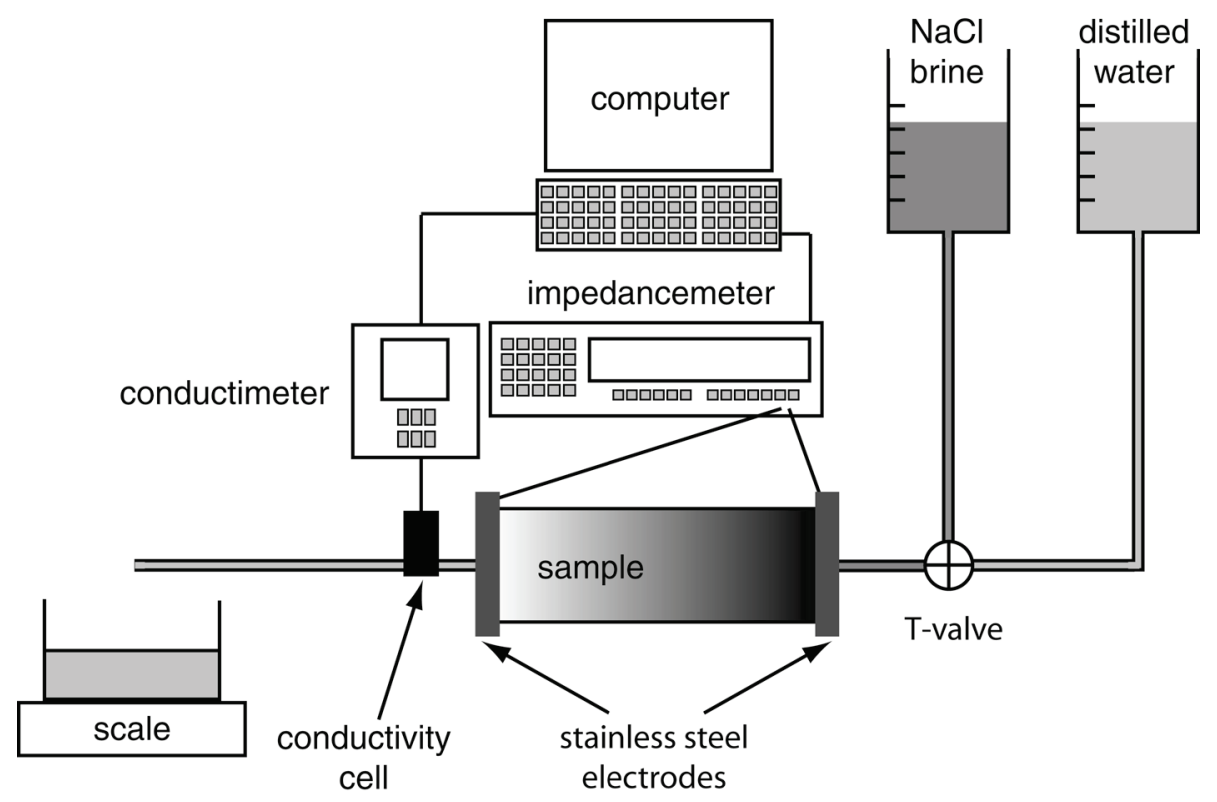

393 Fig. 1. Scheme of the experimental device for miscible displacement. Explanation in the text. 

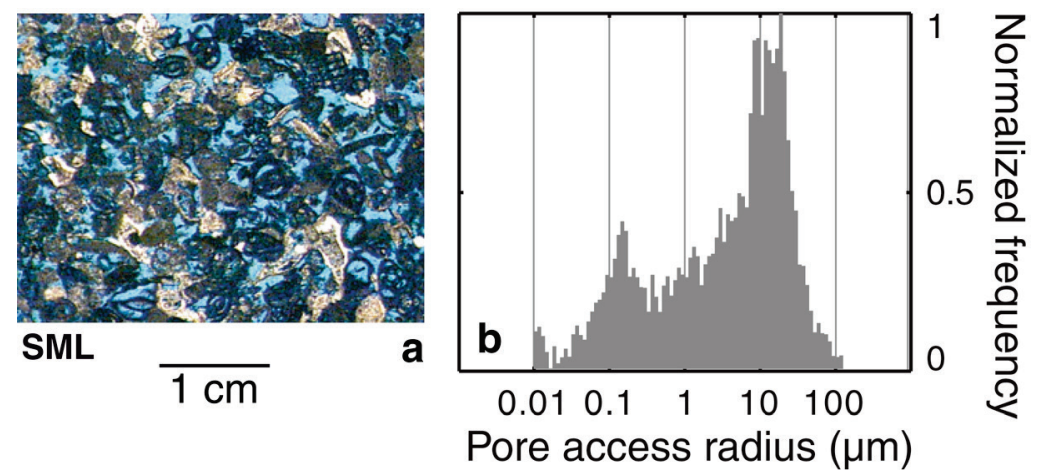

396 Fig. 2. (a) Thin section of Saint-Maximin limestone SML, where the blue areas correspond to the

397 void space filled by the resin; (b) histogram of pore access radius distribution inferred from

398 mercury injection. 

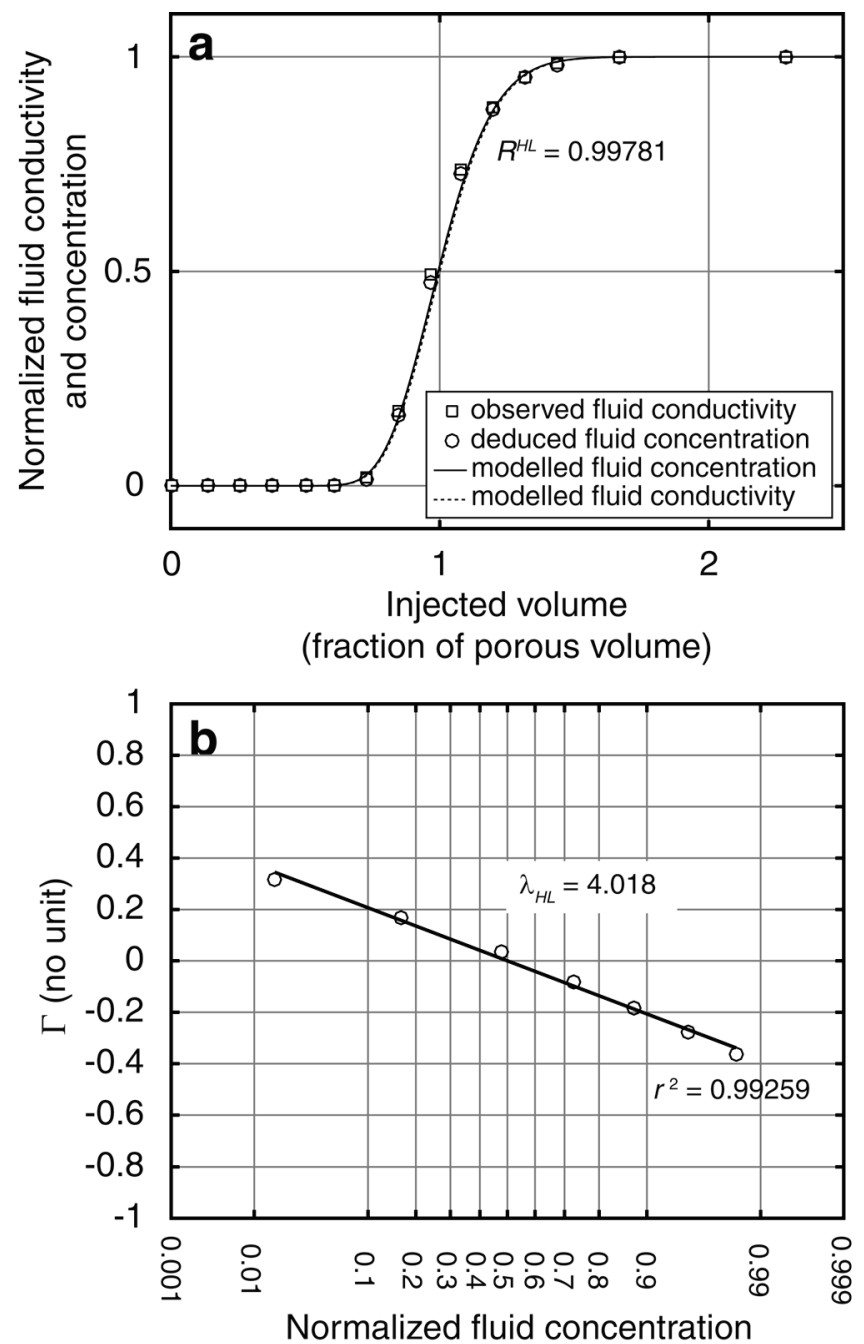

401 Fig. 3. Determination of the dispersivity by fitting the conductivity of the outflowing fluid in

402 Henry's space for Saint-Maximin limestone SML. (a) Observed fluid conductivity and deduced

403 fluid concentration (squares and circles), as a function of the injected volume of brine expressed

404 in fraction of total porous volume; (b) concentration in Henry's space (see text for detail). The

405 dispersivity value $\lambda_{H \mathrm{~L}}=4.018 \mathrm{~mm}$, deduced from the slope of the line, is then used to model the

406 fluid concentration and conductivity shown by the lines in (a). $R^{H L}$ is the value of the cost-

407 function. 

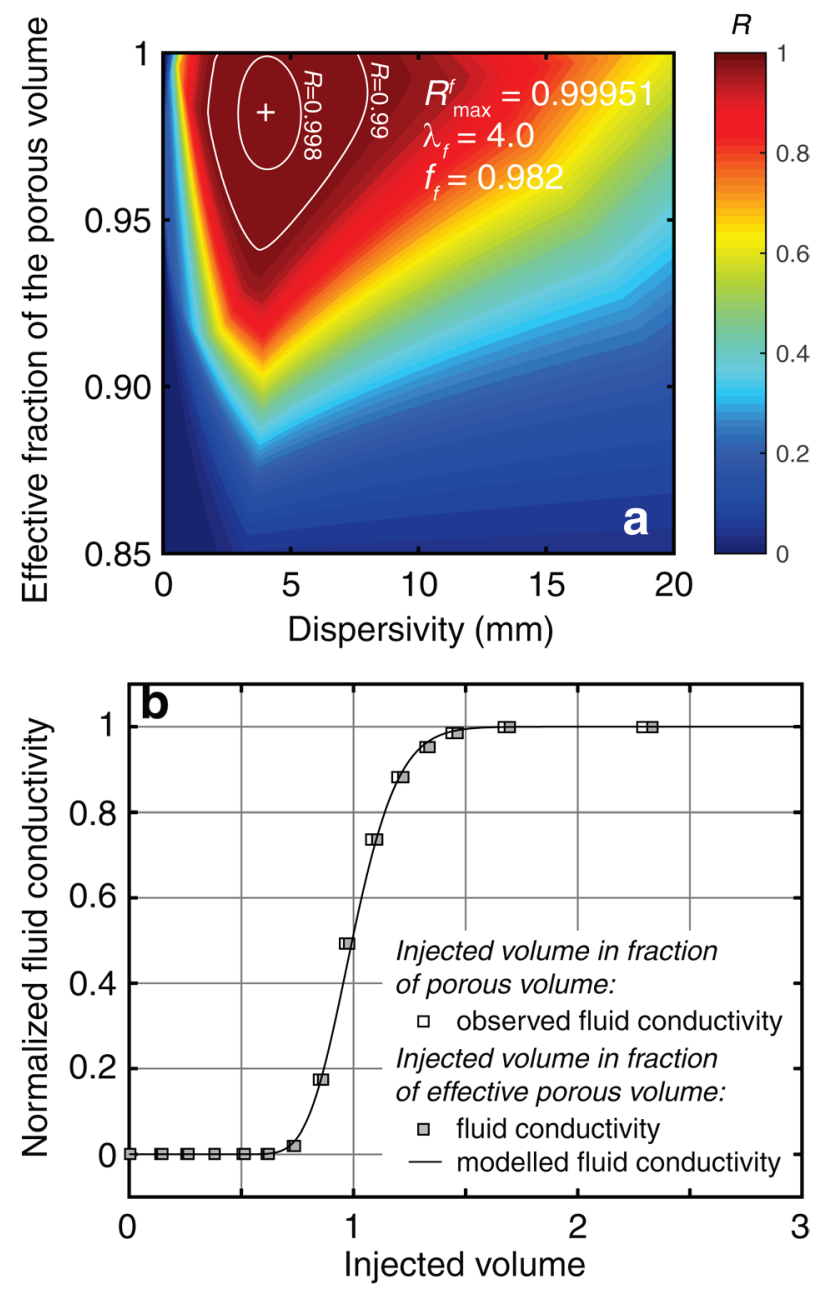

410 Fig. 4. Exploration of the parameter space to model the evolution of the outflowing fluid

411 conductivity for Saint-Maximin limestone SML. (a) isocontours of the cost-function $R^{f}$ as a

412 function of the dispersivity and the effective fraction; (b) optimal solution $\left(\lambda_{f}=4 \mathrm{~mm}\right.$ and

$\left.413 f_{f}=0.982\right)$. 

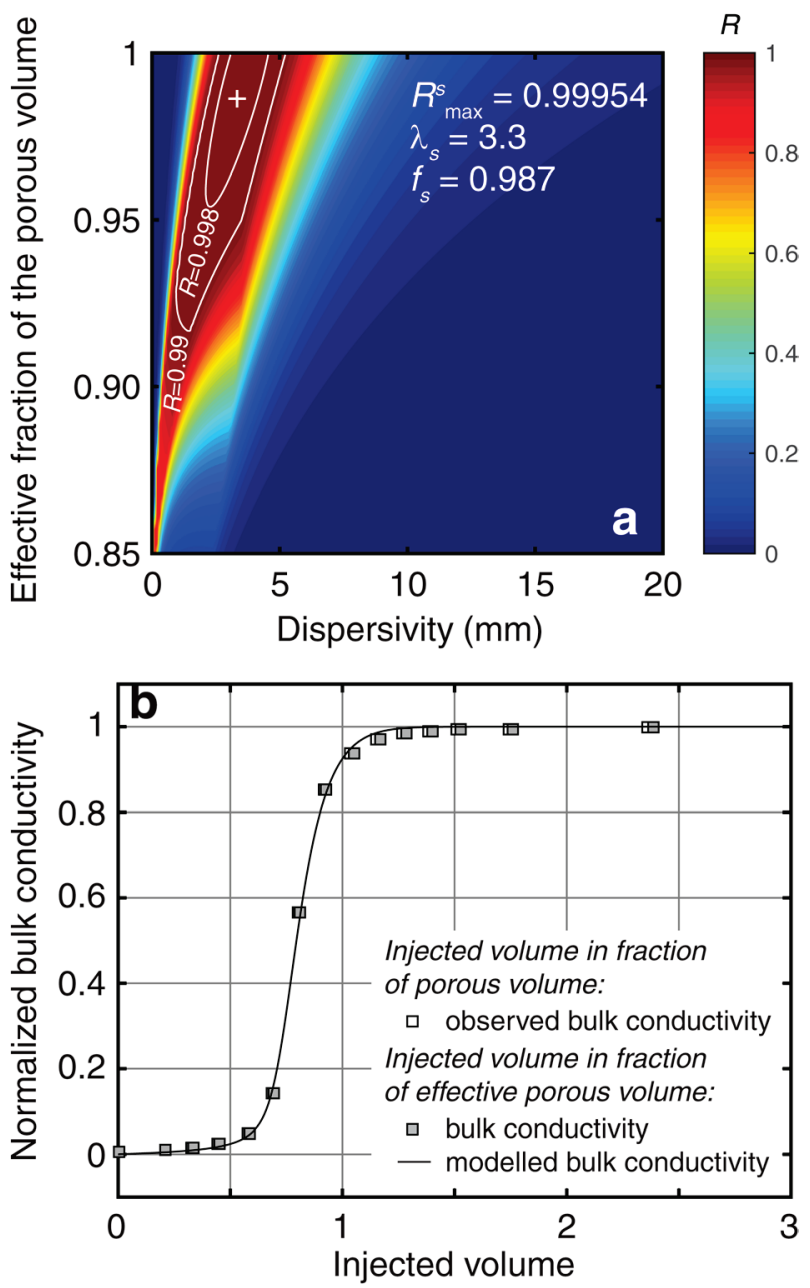

416 Fig. 5. Exploration of the parameter space to model the evolution of the bulk conductivity of the

417 SML sample. (a) isocontours of the cost-function $R^{s}$ as a function of the dispersivity and the

418 effective fraction; (b) optimal solution $\left(\lambda_{f}=3.3 \mathrm{~mm}\right.$ and $\left.f_{f}=0.987\right)$. Note that the cost-function $R^{s}$

419 is more sensitive to the variations of the dispersivity than $R^{f}$, but globally less sensitive to the

420 variations of the effective fraction (compare to Figure 5). 

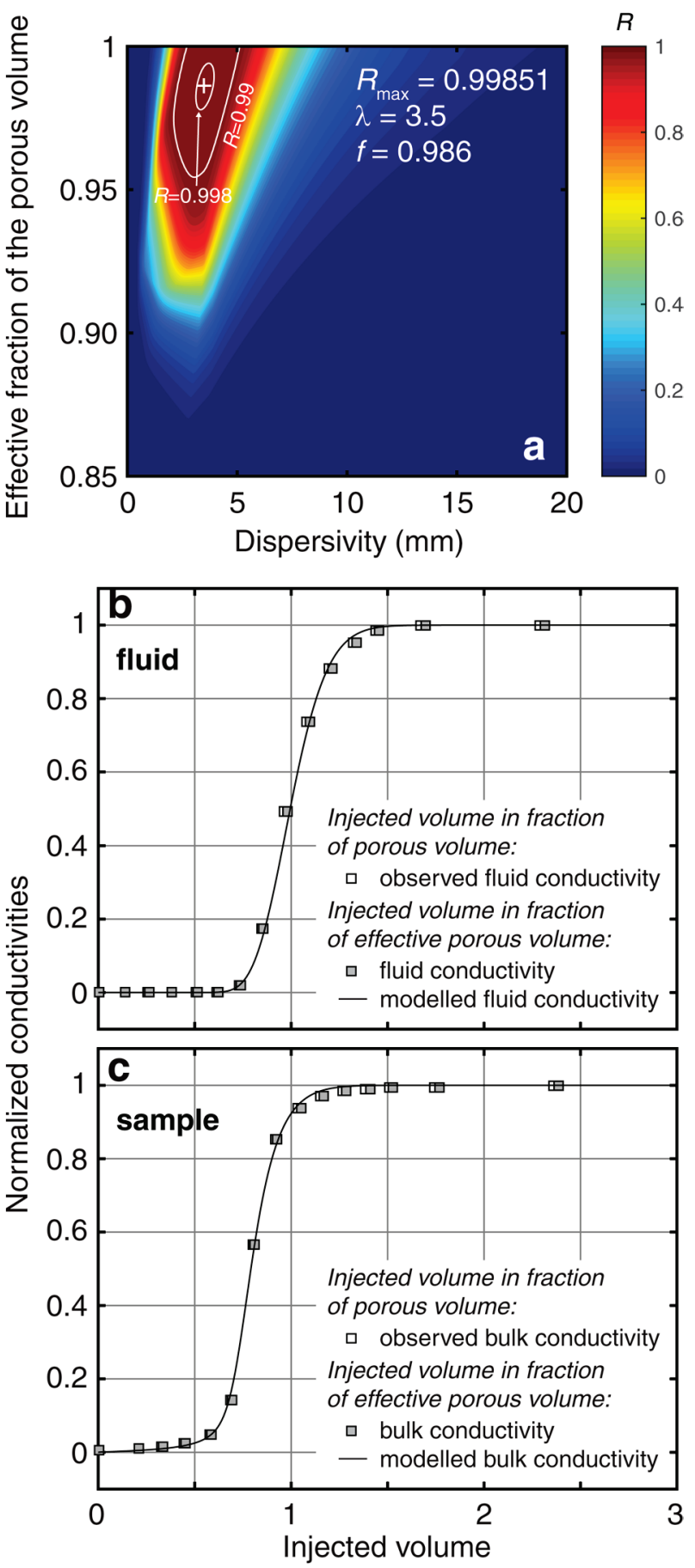

423 Fig. 6. Exploration of the parameter space to model simultaneously the evolutions of the

424 outflowing fluid and bulk conductivities for the SML sample. (a) isocontours of the cost-function

$425 R$ as a function of the dispersivity and the effective fraction; (b) and (c) optimal solution $(\lambda=3.5$

$426 \mathrm{~mm}$ and $f=0.986)$. 